\title{
Mesomechanical Numerical Modeling of the Stress-Strain Localization and Fracture in an Aluminum Alloy with a Composite Coating
}

\author{
Ruslan R. Balokhonov, 2, a), Eugen A. Schwab ${ }^{1, b)}$, Varvara A. Romanova ${ }^{1,2, c)}$, \\ Aleksandr V. Zinoviev ${ }^{1, d)}$, and Sergey A. Martynov ${ }^{1, e)}$ \\ ${ }^{1}$ Institute of Strength Physics and Materials Science SB RAS, Tomsk, 634055, Russia \\ ${ }^{2}$ National Research Tomsk Polytechnic University, Tomsk, 634050, Russia \\ a) Corresponding author: rusy@ispms.ru \\ b) schwab@ispms.ru \\ ${ }^{c)}$ varvara@ispms.ru \\ d) zav@ispms.ru \\ e) martynov@ispms.ru
}

\begin{abstract}
A numerical analysis of plastic strain localization and fracture in an aluminum alloy with a composite aluminum $(\mathrm{Al})$ - titanium carbide $(\mathrm{TiC})$ coating providing oxidation protection is presented. Boundary-value problems in plane strain and three-dimensional formulations are solved numerically by the finite-difference and finite-element methods, respectively. The Al-TiC interface geometry corresponds to the configuration found experimentally and is accounted for explicitly in calculations. An algorithm to build a 3D finite-element model of TiC particles is developed. To simulate the mechanical response of the aluminum substrate and composite coating, use was made of an elastic-plastic model with isotropic strain hardening and a fracture model taking into account crack initiation and growth in the regions experiencing tensile stresses. Local regions of bulk tension are shown to arise near the interfaces even under simple uniaxial compression of the coated material, which controls the mechanisms of plastic strain and fracture localization at the mesoscale level. The role of technological residual stresses is revealed.
\end{abstract}

Keywords: mesomechanics, numerical simulation, coated materials, plastic strain, fracture

\section{INTRODUCTION}

Laser-deposited ceramic composite coatings are widely used in industry to harden material surfaces and prevent their corrosion-erosion and wear. It is found experimentally that residual stresses develop in the surface layer during laser deposition of the coatings [1]. The local stresses may affect the macroscopic strength of the coated materials under mechanical loading.

The main aims of the work are (1) to investigate the mechanisms of plastic strain localization and fracture of the aluminum alloy with a composite coating under uniaxial tension-compression, (2) to simulate the nonhomogeneous stress-strain state in the coating under loading applied to the coating surface and reveal the role of technological residual stresses.

Boundary-value problems are solved numerically by the finite-difference and finite-element methods [2-6]. An elastic-plastic model with isotropic strain hardening and a fracture criterion of the Huber type $[4,6]$ simulate the mechanical responses of the aluminum substrate and ceramic particles, respectively. An explicit account of experimental microstructure of the coated material allows the scale factor in the form of initial conditions to be introduced in the calculations.

International Conference on Physical Mesomechanics of Multilevel Systems 2014

AIP Conf. Proc. 1623, 47-50 (2014); doi: 10.1063/1.4898879

(C) 2014 AIP Publishing LLC 978-0-7354-1260-6/\$30.00 


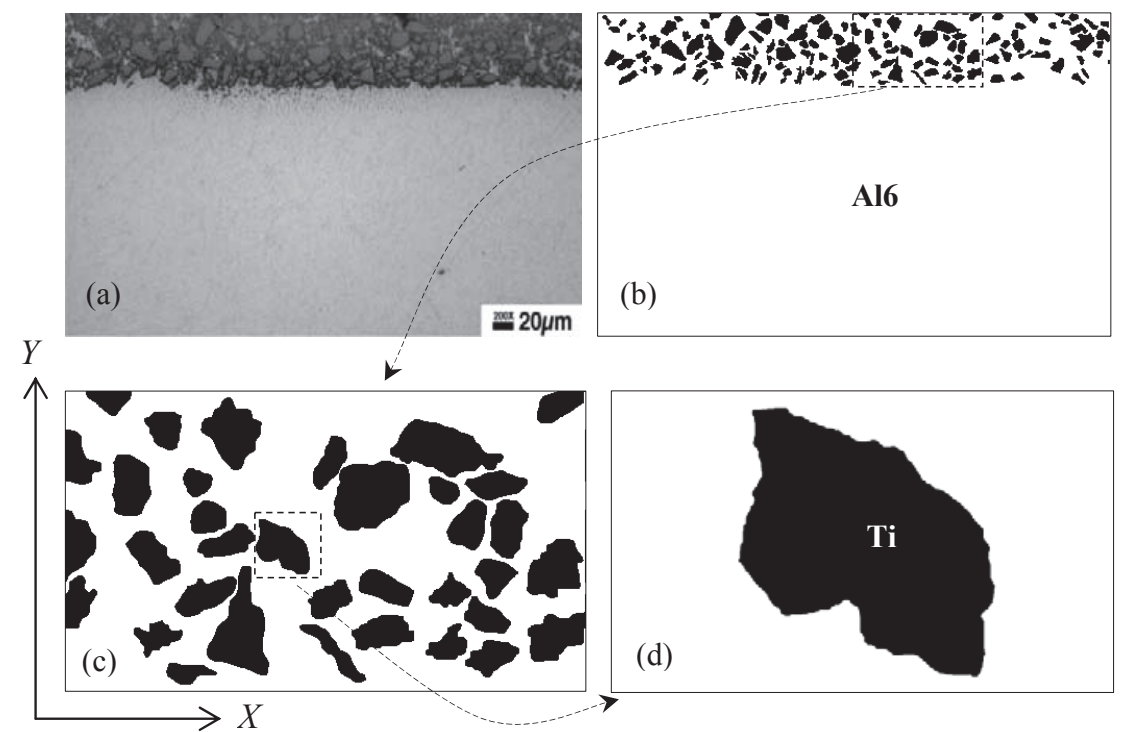

FIGURE 1. Experimental [1] (a) and model microstructures of a material with a composite coating at different scale levels (b)-(d)

\section{RESULTS AND DISCUSSION}

At stage (1) of the investigations, a dynamic problem is solved in the plane-strain formulation [2-6]. Three scale levels are considered: the aluminum substrate with a coating (Figs. 1(a, b)), the composite material of the coating (Fig. 1(c)) and a single particle in the aluminum matrix (Fig. 1(d)). Kinematic boundary conditions on the left and right surfaces simulate uniaxial tension or compression in direction $\mathrm{X}$, while boundary conditions on the top and bottom surfaces are assigned in terms of stresses and correspond to free surfaces. Local regions of bulk tension are shown to arise along the aluminum-titanium carbide interface even under simple uniaxial compression. The tensile regions are located at different points in the cases of external tension and compression, whereas the maximum equivalent stress concentrates in the same local regions.
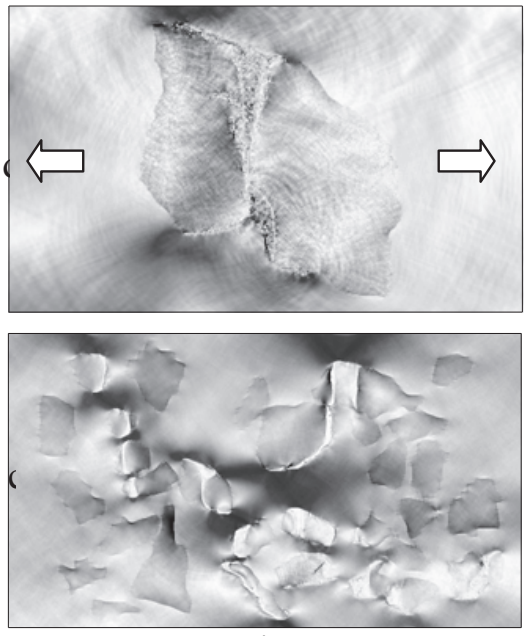

Tension

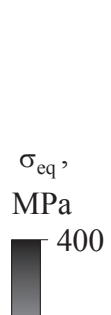

200
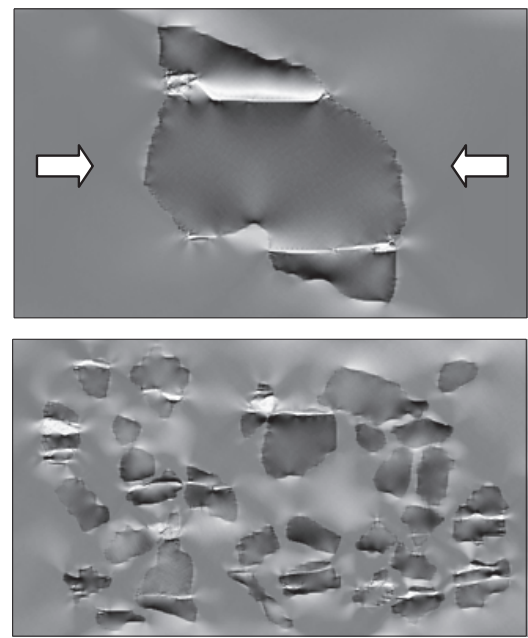

Compression $\sigma_{\text {eq }}$,

$\mathrm{MPa}$

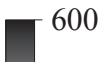

300

FIGURE 2. Cracking of TiC particles in the coating under different types of external loading at scale levels (c) and (d) for the microstructures shown in Fig. 1(c, d). Equivalent stress patterns 

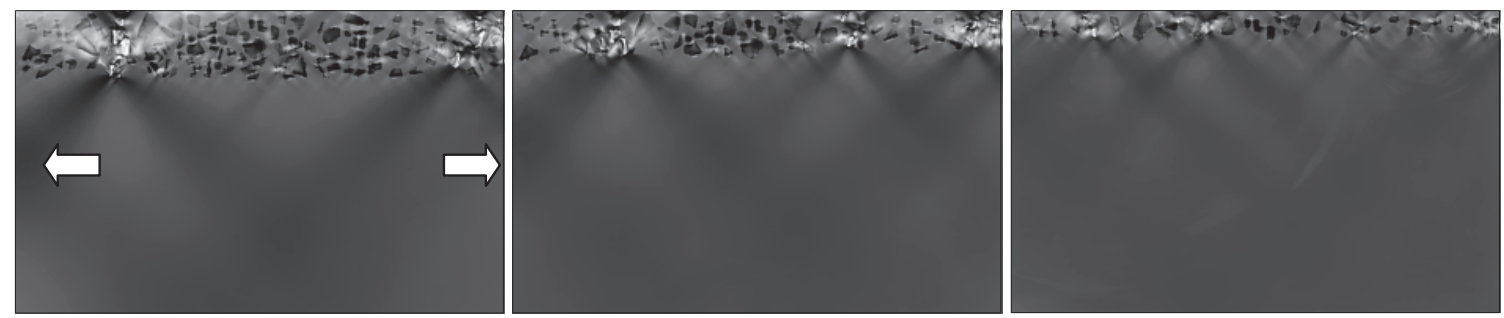

FIGURE 3. Cracking of the TiC-Al composite coating of different thickness

This is valid for different scale levels considered (Figs. 1(b-d)) and controls the mechanisms of fracture localization discussed in this paper. High-strength elastic-brittle ceramic particles are found to rotate in the plastic metal matrix, reducing the stress concentration. As a result, an ultimate compression strength in the local stress concentration regions is never reached, and all the cracks originate and propagate under the action of local tensile stresses both under external tension and compression. Figure 2 presents calculations for coating fragments containing single and several ceramic particles (Figs. 1(c, d)). Cracks under tension and compression are shown to propagate in different directions - perpendicular to and parallel with the loading direction, respectively (Fig. 2). In qualitative terms this fact corresponds to the particle cracking pattern observed experimentally (see, e.g. [7]).

The influence of the coating thickness on the fracture pattern was investigated at scale (b) for the microstructure presented in Fig. 1(b) subjected to tension. The spatial cracking period, i.e. the separation between the cracks formed consecutively in the coating at the right angle to the tensile direction, was found to depend on the coating thickness: the thinner is the coating, the shorter is the separation (Fig. 3). A similar cracking pattern was observed experimentally [8].

Materials are three-dimensional in nature. That is why at stage (2), a 3D boundary-value problem in the quasistatic formulation was solved for more accurate estimation of the stress-strain state of the loaded composite. Use was made of general-purpose software ANSYS based on a finite-element analysis. An explicit account of a 3D material microstructure is not a trivial task. There are layer-by-layer scanning experimental techniques. However, they are very expensive and time-consuming. Another way is to develop model microstructures whose geometric properties are close to those observed experimentally. In this work, a simple method for constructing 3D TiC particles is advanced. Using a natural mechanism of brittle material fragmentation and assuming its scale invariance, we have selected several dozen of rock stones at a mountain quarry. The stone size varied from 80 to $100 \mathrm{~mm}$ in the maximum cross-section. To transform the real shape of stones into a three-dimensional finite-element model, a 3D dynamic video recording of the stone surface was performed, processing the results in real time with a 3DSOM software and storing in the STP format (data transfer standard STEP). The finite-element model of the microstructure was imported in to CAE of the ANSYS solver, using its application Design Modeler. An example of a single particle is shown in Fig. 4 from different angles of view.

Numerical simulations were performed for compression of an aluminum cubic element with the particle shown in Fig. 4. Boundary conditions simulated loading normal to the top surface of the cubic element in direction $Y$. The bottom surface was fixed in direction $Y$, and the left, right, front and back surfaces were load-free.
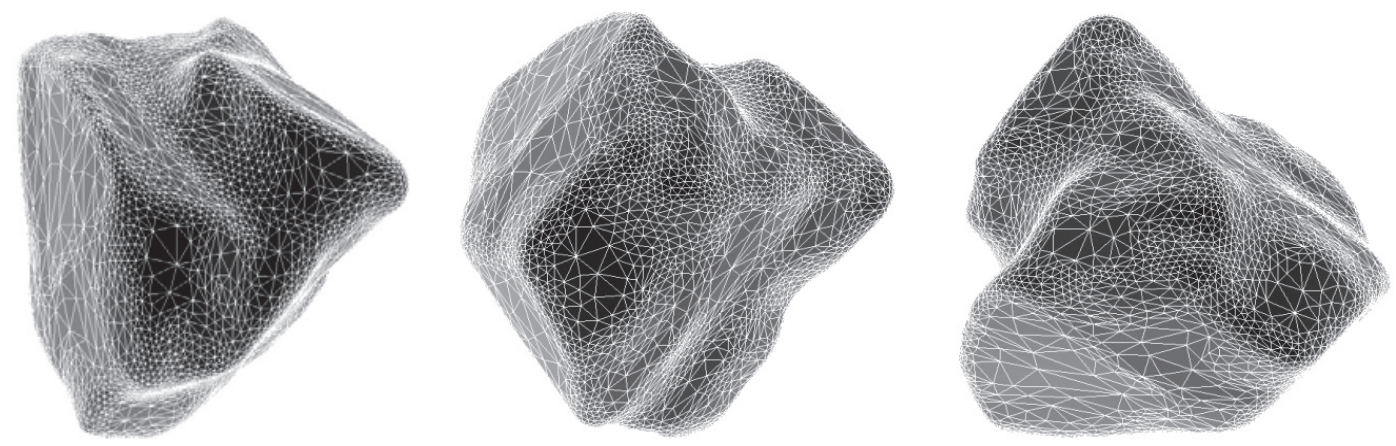

FIGURE 4. Finite-element model of a TiC particle in a composite coating 


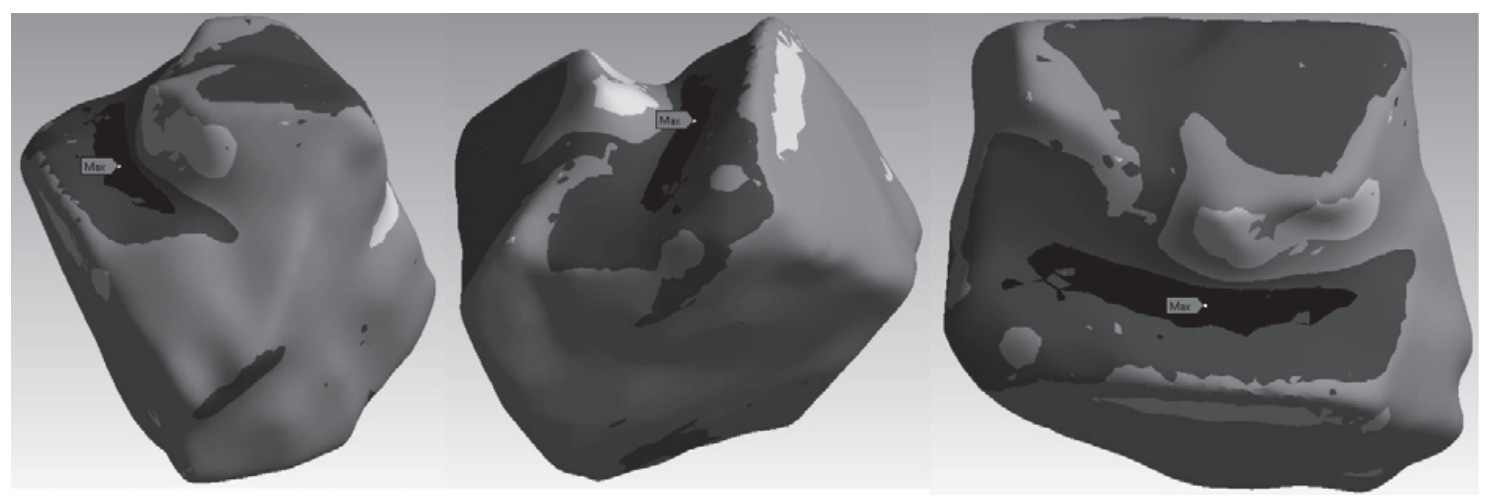

FIGURE 5. Pressure patterns in the TiC particle. White and black colors correspond to positive and negative values, respectively

The numerical experiments have shown that dangerous regions of bulk tension arise in the titanium carbide ceramic particle under compression of the composite (black region with maximum tensile stresses in Fig. 5). It was found experimentally in [1] that the particle and the aluminum matrix experienced compressive and tensile residual stresses, respectively. That is why the compressive residual stresses resist the dangerous mechanical tensile stresses.

Thus, the technological residual stresses formed during the deposition of ceramic composite coatings are shown to be a positive factor further increasing the strength of the coated surface layers under mechanical loading applied to the coating surface.

\section{ACKNOWLEDGEMENTS}

The work was supported by grants from a program to increase the competence of TPU, Russian Foundation for Basic Research (No. 12-01-00436) and Russian Science Foundation (No. 14-19-00766).

\section{REFERENCES}

1. P. B. Kadolkar, T. R. Watkins, J. Th. M. De Hosson, B. J. Kooi, and N. B. Dahotre, Acta Mater. 55(4), 1203 (2007).

2. R. R. Balokhonov, V. A. Romanova, and S. Schmauder, Mech. Mater. 41(12), 1277 (2009).

3. V. A. Skripnyak, E. G. Skripnyak, A. A. Kozulin, V. V. Skripnyak, and M. V. Korobenkov, Russ. Phys. J., 52(12), 1300 (2009).

4. R. R. Balokhonov, V. A. Romanova, S. Schmauder, S. A. Martynov, and Zh. G. Kovalevskaya, Compos. Part B Eng. 66, 276 (2014).

5. Yu. A. Kostandov, P. V. Makarov, M. O. Eremin, I. Yu. Smolin, and I. E. Shipovskii, Int. Appl. Mech. 49(1), 95 (2013).

6. R. R. Balokhonov, V. A. Romanova, S. A. Martynov, E. A. Schwab, Phys. Mesomech. 16(2), 133 (2013).

7. A. Balasundaram, A. M. Gokhale, S. Graham, and M. F. Horstemeyer, Mater. Sci. Eng. A 355, 368 (2003).

8. A. V. Koval and S. V. Panin, Theor. Appl. Fract. Mech. 34, 117 (2000). 